\title{
Principio de precaución en investigación biomédica, seguridad, obligaciones post-investigación y eficacia terapéutica supuesta de las drogas experimentales. Violaciones a la dignidad de los pacientes
}

\author{
The precautionary principle in biomedical research, safety, post-research \\ obligations and alleged therapeutic efficacy of experimental drugs. \\ Violations of patient dignity
}

Gonorazky, Sergio Eduardo ${ }^{1}$

${ }^{1}$ Especialista Consultor de Neurología. Jefe del Departamento de Investigación, Hospital Privado de Comunidad, Mar del Plata, Argentina.egonora@gmail.com

Comentario a: Ugalde A, Homedes N. Cuatro palabras sobre ensayos clínicos: ciencia/negocio, riesgo/beneficio. Salud Colectiva. 2011;7(2):135-148.

Es un lugar común afirmar que un nuevo medicamento debe superar en sus beneficios terapéuticos a los riesgos que conllevan su administración. Sin embargo, desde la elección de la droga que será sometida a estudio experimental hasta el diseño del protocolo, desde su ejecución hasta el análisis de los datos, desde su aprobación por la entidad regulatoria hasta su venta en el mercado, la lógica del lucro de las empresas farmacéuticas y la lógica de las necesidades de nuevos fármacos para la prevención y la atención de enfermedades se encuentran en estado de tensión. En ese sentido, el trabajo de Ugalde y Homedes (1) nos ofrece un abarcativo, interesante, aunque preocupante panorama.

Las drogas experimentales, en tanto sus resultados de eficacia y seguridad son inciertos, deben ser sometidas a estudios en humanos bajo el principio de precaución, aproximación aplicable a la ética del medio ambiente, pero que exige hacerse extensiva a la investigación biomédica.

Expresa Ramón Alcoberro (2), que el concepto de "precaución" no incluye necesariamente una evaluación negativa de la tecnociencia, ni una restricción de la investigación; pero exige una clara conciencia de responsabilidad en todas y cada una de las fases del proceso tecnocientífico. La toma de decisiones, en condiciones de ignorancia e incertidumbre, exige una actitud precautoria, distinta de la preventiva (cuando conocemos los riesgos y sus probabilidades) y de la preventiva cautelar (cuando conocemos los riesgos pero ignoramos sus probabilidades) (3). Por lo conocido y lo informado en el trabajo que motiva este comentario, falta un camino a recorrer para que el principio de precaución se imponga como regla en los ensayos clínicos farmacológicos en humanos.

Un ejemplo de ausencia de aplicación del principio de precaución es la limitada exigencia de las agencias regulatorias respecto a los Comités de Monitoreos de Datos de los Ensayos Clínicos (CMDEC) (4,5), también Ilamados Consejos de Monitoreos de Datos y Seguridad (CMDS) (6) definidos como:

[un] consejo externo que establece el patrocinador para evaluar, a intervalos de tiempo definidos, el progreso de un estudio clínico, los datos de seguridad y los puntos críticos para la evaluación de la eficacia, de manera tal que pueda recomendarle si se debe continuar, modificar o detener un estudio. (7) (cursivas añadidas)

Para la Food and Drug Administration (FDA) los CMDEC son obligatorios solamente en estudios de emergencia en los que no es posible tomar el consentimiento informado, y son solo recomendados en otros como, por ejemplo, estudios grandes, aleatorizados, multicéntricos, cuyo 
objetivo sea prolongar la vida o reducir el riesgo de un evento adverso mayor en la salud, tal como un evento cardiovascular o recurrencia de cáncer. En general, según expresa la $\mathrm{FDA}$, no son requeridos para la mayoría de los ensayos. La Organización Mundial de la Salud (OMS) realiza un extenso enunciado de las situaciones en las que un CMDS puede ser requerido, pero tampoco los plantea como una exigencia. En Argentina, la disposición 6677/10 de la Administración Nacional de Medicamentos, Alimentos y Tecnología Médica (ANMAT) expresa que "el patrocinador puede convocar un consejo independiente de monitoreo de datos" (7), pero al momento actual no lo exige en ninguna situación. Por esta vía, podríamos encontrarnos con ensayos prolongados que busquen aliviar síntomas menores, que tengan consecuencias serias e inesperadas en la rama experimental, en los que, al recaer en forma absoluta el análisis riesgo-beneficio general en el patrocinador, durante el proceso de investigación así como la continuación o terminación del estudio, los análisis y decisiones resulten sesgados.

Por otra parte, la independencia de los CMDEC es controvertida pues a pesar de ser "externos al patrocinador", sus integrantes son nombrados y remunerados directamente por estos. En definitiva, más allá de los controles de seguridad que se implementen en los ensayos clínicos, basados en el principio de precaución, es necesario discutir un mayor nivel de exigencia, autoridad e independencia de los CMDEC.

Ugalde y Homedes comentan críticamente que la industria farmacéutica aduce que genera beneficios por los ingresos en divisas que aportan al país. En ese sentido se expresa claramente la Cámara Argentina de Especialidades Medicinales (CAEME) de Argentina en la revista de la institución de abril de 2010 en un artículo llamativamente titulado "Estudios clínicos, industria sin chimeneas" (8). Podría agregarse que la investigación "enlatada" de los patrocinadores de la industria (en las que el diseño, protocolo y análisis de resultados no los realiza el "investigador") no solo confunde la calidad de investigador con la de reclutador de pacientes, que en numerosas oportunidades distrae recursos humanos que podrían dedicarse a investigaciones basadas en necesidades locales, sino que, en ocasiones, como ocurrió en el estudio COMPAS (señalado por los autores), convierte al profesional en "Cazador de Cuerpos" (9), atentando tanto contra la dignidad de los pacientes como la de los propios facultativos.

Es notable que los patrocinadores y muchos investigadores comenten que los pacientes reclutados para un estudio de investigación obtienen como beneficio una mejor atención médica. Es real que, como producto del proceso de investigación, los pacientes son sometidos a mayores controles, pero si el paciente ya estaba recibiendo una adecuada atención médica, estos mayores controles no otorgan un beneficio adicional a su atención. Por otro lado, si el paciente no estaba recibiendo una adecuada atención médica (como ocurre en poblaciones vulneradas en las que está limitada la accesibilidad a la atención), el paciente recibirá un mayor control durante el estudio, pero su atención mejorará realmente si el investigador se compromete a cumplir la exigencia del artículo 33 de la Declaración de Helsinki 2008 que expresa:

\begin{abstract}
Al final de la investigación, todos los pacientes que participan en el estudio tienen derecho a ser informados sobre sus resultados y compartir cualquier beneficio, por ejemplo, acceso a intervenciones identificadas como beneficiosas en el estudio o a otra atención apropiada o beneficios.

(10) (cursivas añadidas)
\end{abstract}

Lo que significa que el paciente deberá gozar, más allá del resultado del estudio, de la misma accesibilidad beneficiosa declamada por el patrocinador y el investigador luego de finalizar el estudio. Lo contrario, significaría que se instrumentalizó al paciente (afectando su dignidad) por el período que duró la investigación. En la misma revista de CAEME, se comenta:

\footnotetext{
...los primeros beneficiarios de este proceso [de investigación] son los pacientes ya que acceden a tratamientos de última generación que incluso, aún no están disponibles [y que] quienes participan de un estudio clínico tienen acceso a prestaciones de alto estándar de calidad a nivel de intervenciones y atención profesional durante y posterior al desarrollo del estudio. Además existe acceso temprano y prolongado a medicamentos que pueden cambiar por completo el pronóstico de su enfermedad. (8) (cursivas añadidas)
} 
Esta confusión entre tratamiento probado y droga experimental (cuya eficacia terapéutica y efectos adversos se desconocen), de ser transmitida al paciente en el consentimiento informado, resultaría en un engaño que induciría una inapropiada participación de éste en el estudio. La Disposición 6677/10 de ANMAT, en la sección destinada a avisos, expresa que "no deberá indicarse en forma implícita o explícita que el producto en investigación es eficaz y/o seguro o que es equivalente o mejor que otros productos existentes" (7), lo que evidentemente transgrede este escrito de la industria que induce a una manifiesta violación a la dignidad de los pacientes.

\section{AGRADECIMIENTOS}

Agradezco al Consejo Institucional de Revisión de Estudios de Investigación del Hospital Privado de Comunidad (HPC) por haberme dado la oportunidad de discutir estos temas, liberándolos de la responsabilidad de lo aquí manifestado.

\section{REFERENCIAS BIBLIOGRÁFICAS}

1. Ugalde A, Homedes N. Cuatro palabras sobre ensayos clínicos: ciencia/negocio, riesgo/beneficio. Salud Colectiva. 2011;7(2):135-148.

2. Alcoberro R. VORSORGEPRINZIP. El significado del principio de precaución [Internet]. Filosofia i pensament [citado 18 jun 2011]. Disponible en: http://www.alcoberro.info/V1/tecnoetica3.htm

3. Riechmann J. Introducción al principio de precaución [Internet]. Científicos por el Medio Ambiente (CIMA) [citado 18 jun 2011]. Disponible en: http://www.cima.org.es/archivos/Areas/salud_publica/12_saludpublica.pdf

4. Food and Drug Administration. Guidance for clinical trial sponsors establishment and operation of clinical trial data monitoring committees [Internet]. Rockville: FDA; 2006 [citado 18 jun 2011]. Disponible en: http://www.fda.gov/downloads/Regulatorylnformation/ Guidances/ucm127073.pdf

5. Committee for Medicinal Products for Human Use. Guideline on data monitoring committees. London: European Medicines Agency; 2005. EMEA/CHMP/EWP/5872/03 Corr
6. Special Programme for Research and Training in Tropical Diseases (TDR). Operational guidelines for the establishment and functioning of data and safety monitoring boards. Geneva: UNICEF, UNDP, World Bank, WHO; 2005. TDR/GEN/Guidelines/05.1

7. Administración Nacional de Medicamentos, Alimentos y Tecnología Médica. Disposición 6677/2010 [Internet]. 1 nov 2010 [citado 6 jun 2011]. Disponible en: http://www.anmat.gov.ar/Comunicados/Dispo_667710.pdf

8. Estudios Clínicos. Industria sin chimeneas. CAEME. 2010;(1):32-39.

9. Shah S. The Body Hunters: Testing new drugs on the world's poorest patients. New York: The New Press; 2006.

10. Asociación Médica Mundial. Declaración de Helsinki de la AMM. Principios éticos para las investigaciones médicas en seres humanos [Internet]. http://www.wma.net/es/30publications/10policies/b3 /index.html

\section{FORMA DE CITAR}

Gonorazky SE. Principio de precaución en investigación biomédica, seguridad, obligaciones post-investigación y eficacia terapéutica supuesta de las drogas experimentales. Violaciones a la dignidad de los pacientes. [Debate]. Salud Colectiva. 2011;7(2):149-151. 